\title{
Integration of binocular optic flow in cervical neck motor neurons of the fly
}

\author{
Adrian Wertz $\cdot$ Jürgen Haag $\cdot$ Alexander Borst
}

Received: 21 February 2012/Revised: 27 April 2012/ Accepted: 16 May 2012/Published online: 7 June 2012

(c) Springer-Verlag 2012

\begin{abstract}
Global visual motion elicits an optomotor response of the eye that stabilizes the visual input on the retina. Here, we analyzed the neck motor system of the blowfly to understand binocular integration of visual motion information underlying a head optomotor response. We identified and characterized two cervical nerve motor neurons (called CNMN6 and CNMN7) tuned precisely to an optic flow corresponding to pitch movements of the head. By means of double recordings and dye coupling, we determined that these neurons are connected ipsilaterally to two vertical system cells (VS2 and VS3), and contralaterally to one horizontal system cell (HSS). In addition, CNMN7 turned out to be connected to the ipsilateral CNMN6 and to its contralateral counterpart. To analyze a potential function of this circuit, we performed behavioral experiments and found that the optomotor pitch response of the fly head was only observable when both eyes were intact. Thus, this neural circuit performs two visuomotor transformations: first, by integrating binocular visual information it enhances the tuning to the optic flow resulting from pitch movements of the head, and second it could assure an even head declination by coordinating the activity of the CNMN7 neurons on both sides.
\end{abstract}

A. Wertz and J. Haag contributed equally to the study.

A. Wertz · J. Haag · A. Borst

Department of Systems and Computational Neurobiology,

Max-Planck-Institute of Neurobiology, Am Klopferspitz 18,

82152 Martinsried, Germany

Present Address:

A. Wertz (\)

Friedrich Miescher Institute for Biomedical Research,

Structure and Function of Neural Circuits, Maulbeerstrasse 66, 4002 Basel, Switzerland

e-mail: adrian.wertz@fmi.ch
Keywords Motion vision · Insect · Neck motor system · Optic flow $\cdot$ Electrical synapses

\section{Introduction}

For visual orientation and course stabilization, insects rely heavily on the optic flow perceived during flight (Srinivasan and Zhang 2004). In flies, optic flow elicits an optomotor response of the head to stabilize the visual input on the retina (Hengstenberg 1972, 1991; Geiger and Poggio 1977). Thus, optic flow information has to be transformed into an appropriate motor response by a neural circuit.

In blowflies, visual motion information is processed by the well-known lobula plate tangential cells (LPTCs, for review see Borst et al. 2010). The ten cells of the vertical system ('VS cells') and the three cells of the horizontal system ('HS cells') are thought to encode the fly's egomotion as these neurons are tuned to rotations of the fly around different body axes (Karmeier et al. 2003; Krapp and Hengstenberg 1996; Krapp et al. 1998; Wertz et al. 2009a, b). VS and HS cells are the major output elements of the lobula plate (Strausfeld 1976; for review see Borst et al. 2010) and convey the visual motion information to the neck motor system either directly or via descending neurons (Strausfeld and Seyan 1985; Strausfeld and Gronenberg 1990). The neck motor system mediates the head turns with ranges of $\pm 90^{\circ}$ for rotations around the longitudinal body axis ('roll') and $\pm 20^{\circ}$ for rotations around the transverse ('yaw') and vertical ('pitch') axes (Hengstenberg 1991). In blowflies, 21 pairs of neck motor neurons form four known pairs of neck nerves and each neck motor neuron innervates a single muscle (Milde et al. 1987; Strausfeld and Seyan 1985; Strausfeld et al. 1987). Whereas VS and HS cells have mostly monocular receptive 
fields (Krapp et al. 1998; Krapp and Hengstenberg 1996; Wertz et al. 2009b), most neck motor neurons have receptive fields with a higher degree of binocularity (Huston and Krapp 2008). A higher binocularity is useful to differentiate between rotational and translational optic flow (Borst and Weber 2011). Thus, one visuomotor transformational step is to achieve a higher degree of binocularity. For neck motor neurons receiving indirect input from LPTCs, descending neurons could integrate binocular visual motion information (Wertz et al. 2008). However, how neck motor neurons directly postsynaptic to LPTCs gain a higher binocularity is rather unclear.

Here, we focus on two identified neck motor neurons of the cervical nerve (CNMN) which innervate two direct muscles, involved in declining the head (Strausfeld et al. 1987). From anatomical findings, these two CNMNs are thought to be postsynaptic to VS2 and VS3 (Strausfeld and Seyan 1985) and therefore involved in pitch movements of the head. To analyze the visuomotor transformation from LPTCs onto these two CNMNs, we determine the ego-motion tuning of both CNMNs as well as their detailed connectivity to LPTCs and ask the following questions: Can the ego-motion tuning be explained by the connectivity to LPTCs? Do the cells receive input from both eyes? If yes, is the binocular input necessary for an appropriate optomotor response?

\section{Materials and methods}

Preparation and setup

Female blowflies (Calliphora vicina, 2-10 days old, laboratory stock) were briefly anesthetized with $\mathrm{CO}_{2}$ and mounted with wax ventral side up on a small plastic platform. The fly's legs were removed and the wings were immobilized by waxing the base of the wings. For suction electrode recordings of the cervical nerve, the back of the animal was opened from behind and the flight muscles were removed. The cervical nerve was cut with a small pair of iridectomy scissors. For intracellular recordings of lobula plate tangential cells or cervical nerve neck motor neurons in the brain of the fly, the head capsule was opened from behind and trachea and air sacs covering the lobula plate were removed. The proboscis of the animal was cut away and the gut was pulled out. Flies were then mounted on a heavy recording table facing an LED arena. The fly's head and back were viewed from behind through a fluorescence stereoscope (MZ FLIII; Leica, Nussloch, Germany).

Visual stimulation

Visual stimuli were presented on a custom-built LED arena covering $240^{\circ}$ in azimuth and $96^{\circ}$ in elevation of the fly's visual field with an angular separation of $1^{\circ}$ between adjacent LEDs as seen by the fly (for details see Wertz et al. 2009a, b). The spectral peak of the LEDs was at $568 \mathrm{~nm}$. On this LED arena, we presented either global or local stimuli. As global stimuli we used movies corresponding to rotations around various body axes (Wertz et al. 2009a). To generate these movies, we simulated the movements of a fly in a virtual room with checkerboard wallpaper. At every point in time, we projected the environment onto the virtual fly's eye and used the resulting movies subsequently as stimuli. The movies were played on the LED arena with either a sinusoidal or a constant velocity. Movies were shown forward and backward representing a clockwise and counterclockwise rotation. To induce head optomotor responses, we simulated a rotation of the fly around the transverse body axis. However, to determine the preferred rotation of neck motor neurons, we generated a set of movies using different axes of rotation with an interval of $30^{\circ}$. In three dimensions, there are 31 different axes. The resulting movies were played forward and backward resulting in 62 counterclockwise rotations in space. All movies were displayed in a random manner at $180 \mathrm{fps}$ for $500 \mathrm{~ms}$ and $1 \mathrm{~s}$ of pause in between. To map the receptive fields of CNMNs, we applied local stimuli as were previously used to determine the receptive fields of VS cells (Wertz et al. 2009b). Such local stimuli consisted of a bar of $15^{\circ}$ length that was moved across the arena at $120^{\circ} \mathrm{s}$, either horizontally at several elevation angles, or vertically at several points along the azimuth. From the cellular responses to horizontal and vertical bar movement at each location, a vertical and horizontal response component was assigned to this location. The resulting vector points in the cell's preferred direction and the vector length correspond to the cell's motion sensitivity at this location. Such a vector was calculated for 96 spots within the visual field of the fly, with all 96 vectors together representing the receptive field of the cell (Wertz et al. 2009b; Nordström et al. 2008). In the experiment shown in Fig. 7, we divided the arena into different parts. The stimuli had the following dimensions from the origin in front of the fly [azimuth $(\mathrm{az})=0$, elevation $(\mathrm{el})=0$ ]: stimulus $1 \mathrm{az}$ : from $-24^{\circ}$ to $24^{\circ}$, el: from $-16^{\circ}$ to $16^{\circ}$; stimulus $2 \mathrm{az}$ : from $-24^{\circ}$ to $24^{\circ}$, el: from $-48^{\circ}$ to $48^{\circ}$; stimulus 3 az: from $-72^{\circ}$ to $72^{\circ}$, el: from $-48^{\circ}$ to $48^{\circ}$; stimulus 4 : az: from $-120^{\circ}$ to $120^{\circ}$, el: from $-48^{\circ}$ to $48^{\circ}$.

\section{Wind stimuli}

For delivering the wind puffs, we used an air cylinder with a pressure reducer. The wind puffs were directed via tubes to either the antennae or the abdomen of the fly. The flow was triggered by the computer via a solenoid valve (Festo, MHE4-M1H-3/2G-QS-8-K). The air pressure was adjusted to elicit wind puffs with $4 \mathrm{~m} / \mathrm{s}$ wind velocity. 
Measurements of head movement

Flies were anesthetized, fixed with wax on a small glass plate and mounted on the recording table facing the LED arena. A CCD camera (Spot pursuit; Diagnostic Instruments) was used to monitor the head movements of the fly at a rate of 25 frames/s. The stimulus consisted of regularly tiled checkerboard pattern generated as described previously (Wertz et al. 2009a). Stimuli were presented using a sinusoidal velocity profile at a frequency of $0.5 \mathrm{~Hz}$ and a maximum velocity of $300 \%$ s. The acquired movies were analyzed automatically using motion tracking software (Motion Studio; IDT; see also Haag et al. 2010). To analyze the binocular contributions of this head movement, we used the same flies, occluded one eye with ink and repeated the experiment.

Induction and measurements of haltere movements

For inducing active haltere movements by the fly, we directed wind puffs via tubes to the abdomen of the fly. The wind puffs elicited active beating of the halteres in episodes of $0.5-1 \mathrm{~s}$. For measuring the movement of the halteres, we filmed the fly from the side with a high-speed camera (MotionPro Y3, Redlake) with a macro objective (Sigma, $105 \mathrm{~mm} \mathrm{F2,8} \mathrm{EX} \mathrm{DG)} \mathrm{at} \mathrm{a} \mathrm{rate} \mathrm{of} \mathrm{1,000} \mathrm{frames/s}$ (Fig. 9). The computer triggered the acquisition and the acquired movies were tracked with a custom-written program in Matlab.

\section{Electrical recordings}

For intracellular and nerve-suction recordings, glass electrodes were pulled (Flaming/Brown micropipette puller, P-97; Sutter Instrument, Novato, CA) using glass capillaries with an outer diameter of $1 \mathrm{~mm}$ (GC100F-10; Science Products, Hofheim, Germany). Electrodes had resistances between 25 and $50 \mathrm{M} \Omega$. For suction electrode recordings, glass electrodes were scored with a diamondtipped "pencil" under a microscope, and the tip was broken at the score. The diameter of the resulting electrode had approximately the size of the cervical nerve. For intracellular recordings, the tip solutions contained either $10 \mathrm{mM}$ Alexa 488 or 10 mM Alexa 594 (both Invitrogen, Carlsbad, CA). For dual intracellular recordings from two cells, one electrode was filled with the green fluorescent dye Alexa 488 , and the other one with the red fluorescent dye Alexa 568 (Molecular Probes). For neurobiotin staining, the tip of the electrode was filled with a mixture of $3 \%$ neurobiotin (Vectorlabs) and $3 \%$ fluorescein (Sigma). For all intracellular recordings, the shaft of the electrode was filled with $2 \mathrm{M}$ potassium acetate plus $0.5 \mathrm{M}$ potassium chloride. For data analysis, the output signals of the SEL10-amplifier (npi electronics, Tamm, Germany) operating in bridge mode were fed to a $\mathrm{PC}$ via an $\mathrm{A} / \mathrm{D}$ converter (PCIDAS6025, Measurement Computing, Massachusetts, USA) at a sampling rate of $10 \mathrm{kHz}$ for intracellular recordings, $30 \mathrm{kHz}$ for extracellular recordings, and $5 \mathrm{kHz}$ for dual intracellular recordings. An additional SEL10 amplifier was used for dual intracellular recordings. For intracellular recording from motor neurons of the cervical nerve (CNMNs), we first filled the cell with a fluorescent dye from its clearly visible soma. We then inserted the electrode in the fluorescently labeled processes to record the cell at either its dendrite or its axon. Whereas recordings from the cell bodies of CNMNs were rather stable allowing recordings for up to $30 \mathrm{~min}$, recordings from the dendrites and axons were limited to $10 \mathrm{~min}$ the most. After an intracellular recording, several images of each Alexa-filled cells were taken by a CCD camera (Leica DC 320, Bensheim, Germany). The anatomy of CNMN was imaged in a two-photon microscope (see below). These images allowed anatomical identification of the recorded cells on the basis of their characteristic branching patterns (for CN-NMNs: Strausfeld et al. 1987) and the relative position of their ventral dendrite within the lobula plate. Software for stimulus control, data acquisition, spike sorting by spike characteristics and data analysis was programmed in Matlab (Mathworks). Neural responses were determined by either counting the spikes $100 \mathrm{~ms}$ after stimulus onset until the end of the stimulation minus the mean resting frequency within $200 \mathrm{~ms}$ before stimulus onset or, for graded responses, by taking the average membrane potential for the same time intervals. For the multiunit recordings, we analyzed the data in Matlab either with a custom-written program or with Waveclus, a fast and unsupervised algorithm for spike detection and sorting (Quiroga et al. 2004). The custom-written program used a threshold to detect the spikes and the $k$-mean algorithm of Matlab to cluster the spikes according to their maxima and minima. Both the custom-written program and the Waveclus software led to similar results.

\section{Two-photon microscopy}

We used a custom-built two-photon microscope (Denk et al. 1990; Haag et al. 2004) consisting of the following components: a 5-W pumped Ti:Sapphire laser (MaiTai; Spectra Physics), a Pockels cell (Conoptics), scan mirrors with drivers (Cambridge Technology), a scan lens (4401302; Rodenstock), a tube lens (MXA 22018; Nikon), a dichroic mirror (DCSPR 25.5 - 36; AHF Tuebingen) and a $40^{\circ}$-water immersion lens (Zeiss). The lens can be moved along all three axes by a step motor-driven micromanipulator (MP285-3Z; Sutter Instruments). Emitted light is filtered in parallel by two bandpass filters (HQ 535/50M and 
HQ HQ610/75M; Chroma) and collected by multialkali photomultipliers (R6357; Hamamatsu). The whole system is controlled by custom-written software (CfNT V.1569 developed by Winfried Denk and Michael Mueller, MaxPlanck Institute for Medical Research, Heidelberg).

\section{Histology}

After filling a cell with the neurobiotin and fluorescein mixture, it was identified under the fluorescence microscope. The fly was then kept at $+4^{\circ}$ for at least $60 \mathrm{~min}$ to allow for diffusion of neurobiotin to coupled cells. For streptavidin staining, brains were fixed in $4 \%$ paraformaldehyde $+0.2 \%$ glutaraldehyde (60 min to overnight), washed for 45-60 min in phosphate-buffered saline (PBS) including $2.5 \%$ Triton $\mathrm{X}-100(\mathrm{pH} 7.4)$, and then for $10 \mathrm{~min}$ in PBS including $1 \%$ Triton $\mathrm{X}-100(\mathrm{pH} \mathrm{7.4)}$ ). Streptavidin Alexa Fluor-568 conjugate (Invitrogen) was added at a ratio of $1: 100$ overnight $\left(4{ }^{\circ} \mathrm{C}\right)$. The stained brains were mounted in GEL-MOUNT (Science Services) and analyzed by confocal microscopy.

\section{Electron microscopy}

Before dissection of the fly brain and the thoracic ganglion, the fly was fixed in paraformaldehyde for 30-60 min. After dissection, we left the tissue in paraformaldehyde overnight. After washing in $0.1 \mathrm{M}$ sodium cacodylate (Sigma), brains were incubated in $1 \%$ osmium tetroxide (Science Services) for $15 \mathrm{~min}$ and afterward dehydrated with an ethanol series in the following steps: 30, 50, 70, 90, 96, 2× $100 \%$ ethanol. Propylene oxide (Serva) was then used to remove the residual ethanol $(2 \times 10 \mathrm{~min})$ before the tissue was embedded in Epon (Serva) and polymerized at $60^{\circ}$ for 2 days. Ultrathin sections of $60 \mathrm{~nm}$ were cut with the Ultra microtome Leica EM UC6i (Leica Microsystems) at distinct positions of the cervical nerve. For counterstaining, sections were incubated in lead citrate and $0.5 \%$ uranylacetat (Ultrastainer, Leica Microsystems) in grids coated with formvar (Science Services), and then analyzed with the electron microscope (JEM-1230, Jeol).

\section{Results}

In the first set of experiments, we identified the neck motor neurons of interest involved in head declination. Neck motor neurons of the cervical nerve (CNMNs) are thought to be key players in pitch movements of the head (Strausfeld et al. 1987). These neurons originate in the brain, descend the connective and leave it to form the cervical nerve (Fig. 1a). To determine the exact number of neurons within the nerve, we imaged cross sections of the cervical nerve with an electron microscope (Fig. 1b). In all three flies examined, we found ten axons running through the cervical nerve on either side (Fig. 1b). Based on cobalt fillings, Strausfeld et al. (1987) identified eight motor neurons within this nerve having either unilateral or bilateral connections. To analyze how many of these CNMNs are sensitive to pitch movements, we performed suction electrode recording of the whole nerve (Fig. 1c). While presenting pitch upward and downward movements on the LED arena, different spike shapes were observed, which could then be sorted off-line according to the maxima and minima of their waveforms (Fig. 1d, e). In the example shown, we found five out of the ten neurons responding to the pitch stimulus and thus to be motion sensitive. In detail, units 1 and 2 showed an increase of firing frequency to a nose-up pitch movement and no response to nose-down pitch movement. Units 3 and 4 also responded with an increased firing rate to nose-up pitch movement, but in addition revealed a slight excitation in response to nosedown pitch movement. In contrast to all other units, unit 5 showed an opposite preference, i.e., an increase of firing frequency to nose-down pitch movement and a weak response to nose-up movement. In all our suction electrode recordings $(n=21)$, we reliably found at least one unit producing larger spikes (indicated by the red arrow) and one unit responding with a strong increase in firing frequency to the nose-up pitch stimulus (indicated by the blue arrow). To identify the respective neurons, we performed double recordings. We recorded the $\mathrm{CN}$ response with a suction electrode as before and searched for the neurons of interest with a sharp electrode. From the anatomical description of CNMNs from Strausfeld et al. (1987), we expected the cell bodies of CNMN6 and CNMN7 in the protocerebrum next to the esophagus foramen. We found at least three of the CNMNs, which have their cell bodies beneath the esophagus on one hemisphere and their axon running on the opposite hemisphere. In the following, we refer to the hemisphere where the cell body is located as ipsilateral and the hemisphere where the axon is running as contralateral. In the example shown (Fig. 2a, b), two CNMNs were filled with Alexa 488 or Alexa 568, respectively, and were reconstructed after imaging the cells' anatomy with a two-photon microscope. Both cells have arborizations in both hemispheres and resemble the cervical neck motor neurons 6 and 7 described by Strausfeld et al. (1987). Accordingly, we refer to the green cell as CNMN6 and to the red cell as CNMN7. Simultaneous intra- and extracellular recording allowed us then to assign the units as recorded by the suction electrode to CNMN6 and CNMN7 (Fig. 2c, e). In addition, current injection of $\pm 10 \mathrm{nA}$ into the cell's dendrite or cell body increased or decreased the firing frequency of the cell, respectively (Fig. 2d, f). We found that CNMN6 and CNMN7 resemble 

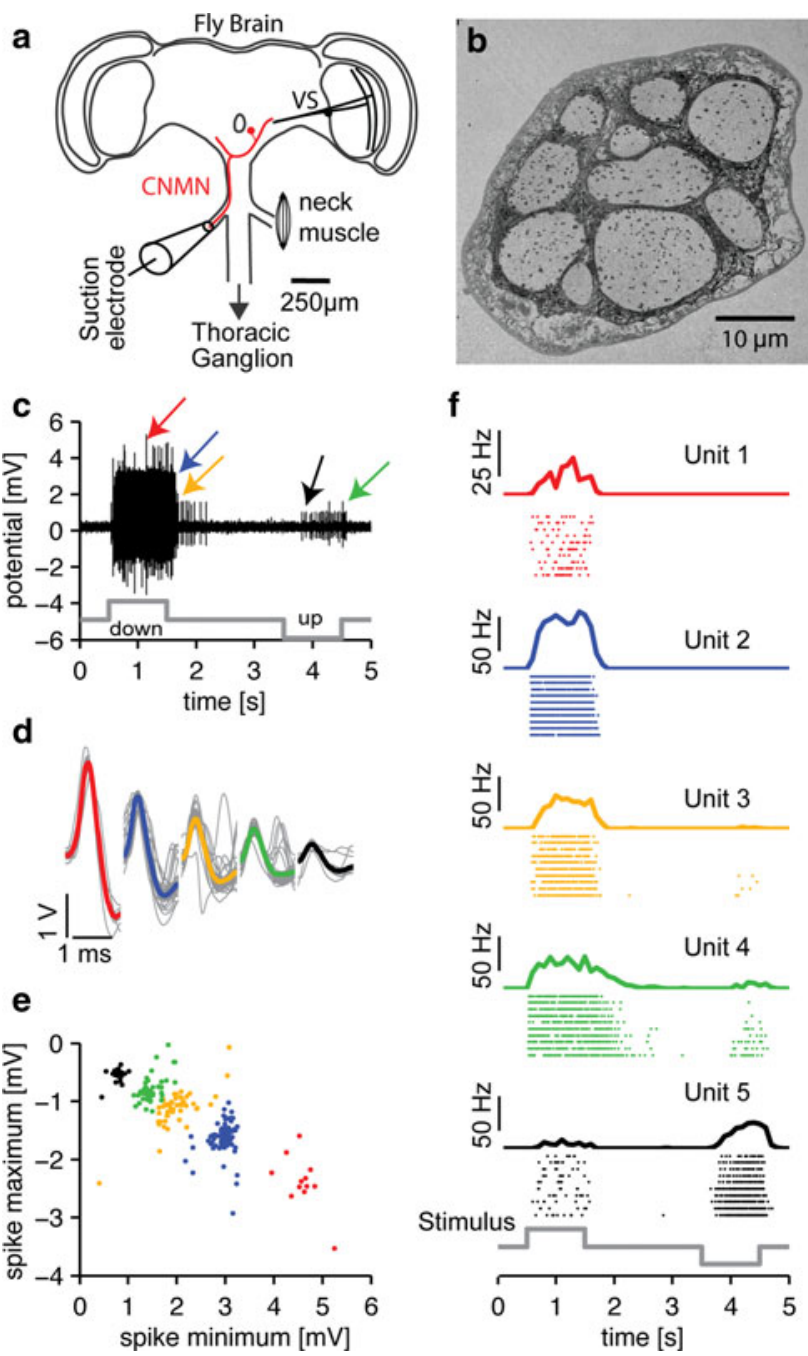

Fig. 1 Suction electrode recording of the cervical nerve (CN). a Schematic drawing of the investigated neural network with a suction electrode at the position where the $\mathrm{CN}$ emerges from the connective. b Example of an electron microscope image showing a $\mathrm{CN}$ cross section containing ten neurons. c Example of suction electrode recording from the $\mathrm{CN}$ while a nose-down and nose-up pitch was presented with a sinusoidal velocity. d Averaged spike shapes of five units responding to visual motion. Units could be sorted according to their spike shape (d, e and arrows in c). e Cluster plot of the spike minima and maxima of different units. $\mathbf{f}$ Rasterplot and peristimulus-time histograms of the units to a nose-down and nose-up pitch. Each unit responded to nose-up pitch, whereas nose-down pitch elicited only a response in units 4 and 5. Mean response of ten sweeps

units 2 and 3 shown in Fig. 1. In addition, both cells project to the ventro-longitudinal muscle responsible for head declination (Strausfeld et al. 1987).

To analyze which ego-motion these cells are tuned to, we determined the preferred axis of rotation of these CNMNs. Example responses of CNMN7 intracellular recordings to the cardinal axes are shown in Fig. 3a. The cell depolarized to one direction (preferred direction) and hyperpolarized to the opposite one (null direction). In the
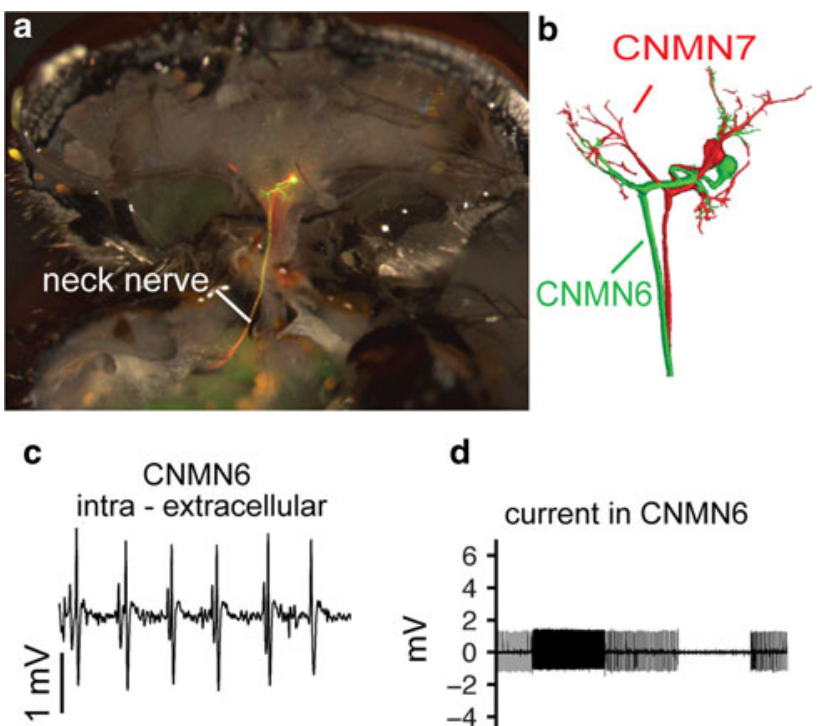

d
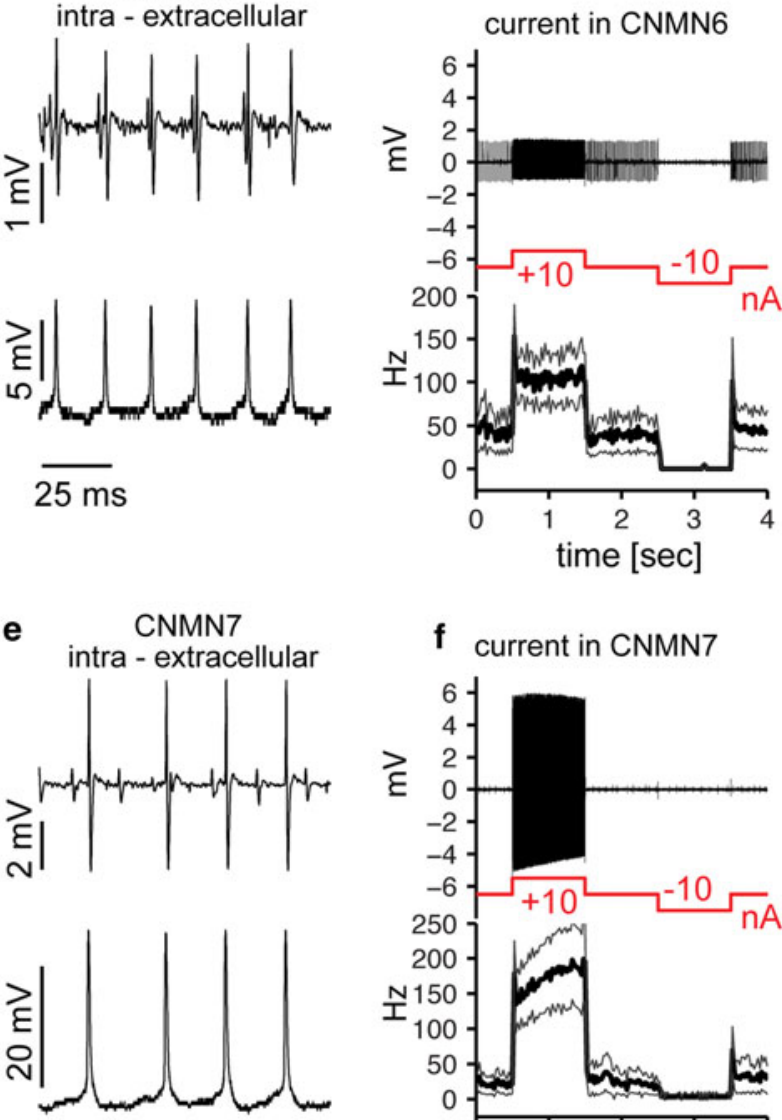

$25 \mathrm{~ms}$

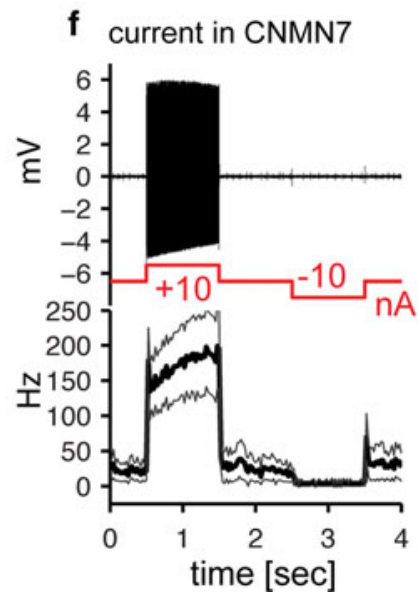

Fig. 2 Extra- and intracellular recording of CNMN6 and CNMN7. a In this example, CNMN6 (green) and CNMN7 (red) were filled with Alexa 488 or Alexa 594, respectively. b The reconstructions of both cells revealed the arborizations in both hemispheres and the axons contralateral to the cell bodies. c Example of a simultaneous intracellular recording of CNMN6 (lower trace) and a suction recording (upper trace). d Example of trace and mean response \pm SEM (3 flies) to positive and negative current injection into CNMN6. e Example of a simultaneous intracellular recording of CNMN7 (lower trace) and a suction recording (upper trace). f Example of trace and mean response \pm SEM (6 flies) to positive and negative current injection into CNMN7. Positive current into CNMN6 or CNMN7 elicited spikes in the suction electrode recording. Current injection into CNMN7 elicited spikes with a higher amplitude (compare $\mathbf{d}$ and $\mathbf{f}$ ) allowing an assignment of the suction recording to a cell 

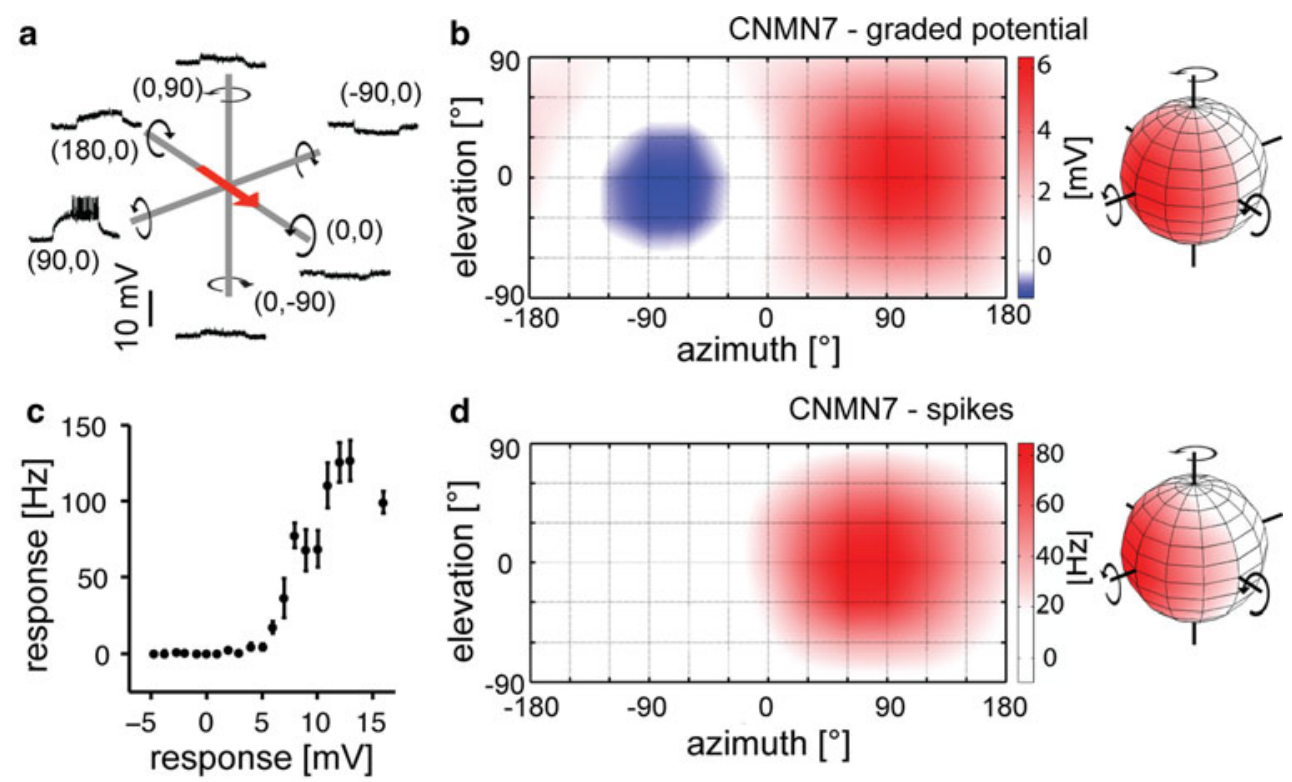

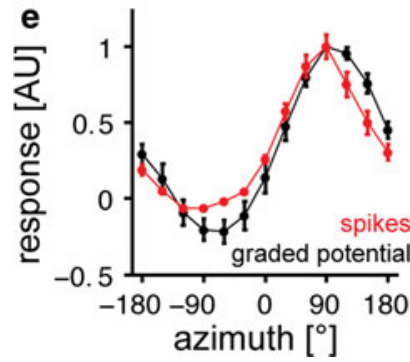

Fig. 3 Rotational action fields of CNMN6 and CNMN7. a Intracellular recording in the cell body of a CNMN7 cell to rotations around the $x$-, $y$ - and $z$-axis. The arrow indicates the position of the fly, and the direction of the arrow the orientation the fly's head. b Mercator and sphere representation of the rotational action field of CNMN7 calculated from the graded response of the cell. Red represents a depolarization of the cells, blue a hyperpolarization. Each square in the Mercator map represents an axis of rotation. The cell is tuned to nose-up pitch movement. c Spike versus graded response of CNMN7

mercator and spherical plot, the mean graded responses of CNMN7 to all 62 rotations were shown color-coded with red representing a depolarization and blue a hyperpolarization (Fig. 3b). The coordinates $(0,0)$ represent the roll axis $(90,0)$, pitch axis and $(90,90)$ yaw axis. Since these responses reflect a particular rotational action of the animal, we call these response fields 'rotational action fields', as introduced by Borst and Weber (2011). CNMN7 responded strongest to a rotation around the pitch axis. To analyze whether the spike threshold affects the tuning of CNMN7, we first determined the membrane potential change necessary to elicit spikes in the cell (Fig. 3c). Therefore, we used all responses induced by visual stimuli and plotted the spike rate as a function of the membrane potential change. It turned out that a depolarization of more than $5 \mathrm{mV}$ was necessary to elicit action potentials. With increasing depolarization, the spike rate rose quickly. to visual stimulation. A depolarization of more than $5 \mathrm{mV}$ elicited spikes in the cell. d Mercator and sphere representation of the rotational action field of CNMN7 calculated from the spiking response. Both graded as well as spiking response of CNMN7 indicates a similar tuning of the cell, also shown in the equatorial tuning curves of CNMN7. e CNMN7 is tuned to a nose-up pitch rotation ( $n=4$ flies). $\mathbf{f}$ Mercator and sphere representation of the rotational action field of CNMN6 calculated from the graded potential response $(n=1$ fly $)$

Second, we calculated the rotational action field of CNMN7 from the spiking response (Fig. 3d). Comparing these rotational action fields, only slight differences were observable. First, in the spiking rotational action field, the hyperpolarizing response was not observable. Second, the rotational action field of the spiking response was slightly shifted toward the origin $\left(\mathrm{az}=0^{\circ}\right.$, el $\left.=0^{\circ}\right)$. Third, the rotational action field from the graded response showed a broader tuning around the pitch axis than the rotational action field of the spiking response. This difference was better observable when plotting the equatorial tuning curves of the spiking and graded responses (Fig. 3e). However, both rotational action fields showed a clear tuning of the cell to a nose-up pitch movement, indicating that a spike threshold does not influence much the tuning of the cell. CNMN6 showed a similar tuning as CNMN7 (Fig. 3f), although the presented visual stimuli elicited no 


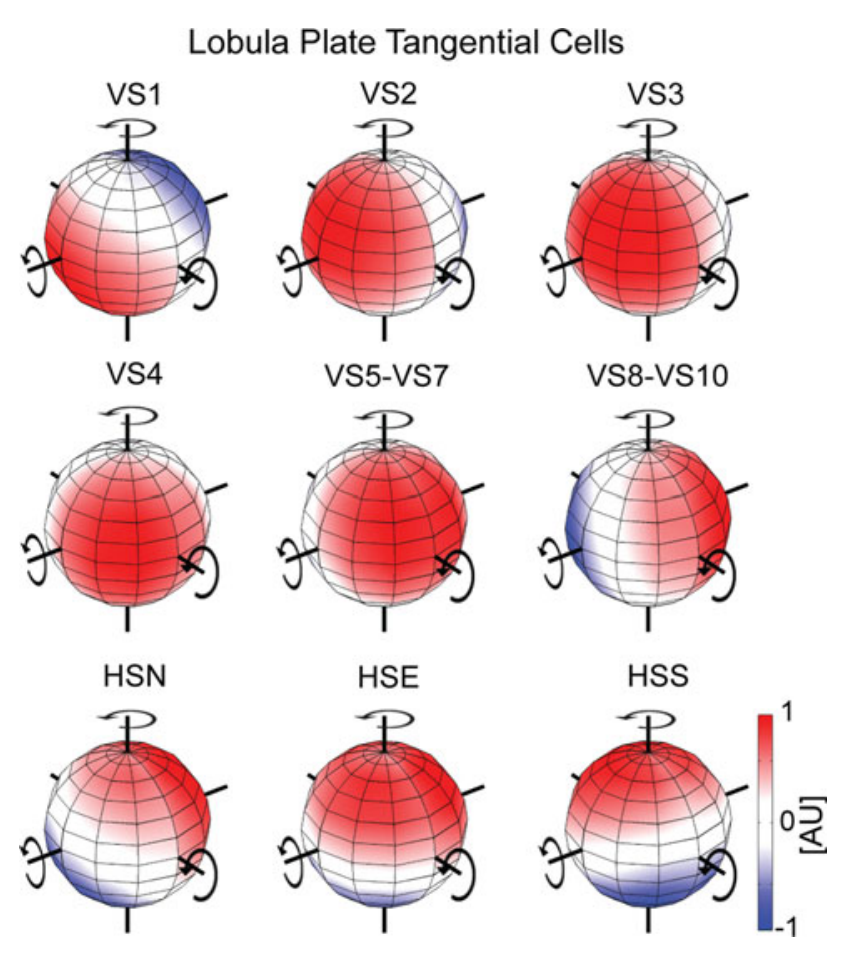

Fig. 4 Rotational action fields of LPTCs. Red represents a depolarization of the cells, blue a hyperpolarization. Mean response from $n$ flies (VS1 $n=2$, VS2 $n=3$, VS3 $n=3$, VS4 $n=2$, VS5-VS7 $n=5, \operatorname{VS} 8-\operatorname{VS} 10 n=2, \operatorname{HSN} n=2, \operatorname{HSE} n=1, \operatorname{HSS} n=2$ )

spiking in CNMN6. In Fig. 2a, pitch stimulus elicited spikes in CNMN6, which was probably due to a depolarization of the cell, caused by the suction recording. For CNMN6 as well as for CNMN7, the absolute graded responses to nose-down pitch were less strong compared to the response to nose-up pitch.

To obtain an impression which cells provide the input to CNMN6 and CNMN7, we determined the preferred axis of rotation from potential presynaptic cells in the same way (Fig. 4). We recorded the responses of the well-described cells of the vertical (VS1-VS10) and the horizontal system (HSN, HSE and HSS). From these response profiles, VS2 and 3 appear to have similar rotation tuning as CNMN6 and 7. Therefore, it is most likely that CNMN6 and CNMN7 receive input from lobula plate cells VS2 and VS3, as proposed by Strausfeld et al. (1987) based on cobalt coupling. In the next set of experiments, we determined the connectivity of CNMN6 and CNMN7 anatomically as well as physiologically. First, we injected neurobiotin into the CNMNs. Neurobiotin is a small molecule and was shown to cross electrical synapses in fly neurons (Haag and Borst 2005; Joesch et al. 2008). CNMN7 was dye coupled to ipsilateral VS2 and VS3 cells, the contralateral HSS cell and at least one ipsilateral neuron, which as probably ascending as no soma was observable in the brain (Fig. 5a). In addition to these strong dye couplings, the ipsilateral CNMN6 and
CNMNs from the opposite side were more weakly labeled. Neurobiotin injection into CNMN6 resulted in a weak dye coupling to VS cells (Fig. 5b). To confirm the dye coupling, we injected neurobiotin into the VS2 and VS3 (an example of neurobiotin injection into VS3 is shown in Fig. 5c) and HSS (Fig. 5d). In all three cases, we found a dye coupling to CNMN7, suggesting an electrical coupling between these cells. Interestingly, we found a labeling of the ipsilateral and contralateral somas of CNMNs on injecting the neurobiotin into CNMN7, VS2, VS3 or HSS cells (indicated by an asterisk in Fig. 5c, d).

To confirm the results of the dye coupling, we performed double intracellular recordings of VS- and HS cells and CNMNs. Therefore, we recorded the CNMNs in the dendrite and the lobula plate neurons in the axon terminal region and injected a current of $\pm 10 \mathrm{nA}$. However, positive or negative current injection into a presynaptic cell elicited only a small membrane potential change $(<0.5 \mathrm{mV})$ in CNMNs. Thus, for an analysis of the connectivity, we depolarized the CNMNs (1.5-2 nA) until the cells showed a resting spike frequency of about $20-40 \mathrm{~Hz}$ (Fig. 5e). Under these conditions, CNMN6 and CNMN7 increased and decreased their firing rate to positive and negative current injection into the ipsilateral VS2 and VS3 (Fig. 5f, g), indicating electrical synapses between them. Current injection into ipsilateral VS1 or VS4 elicited only a small response in CNMNs, which is probably due to the chain-like, electrical coupling between VS cells (Haag and Borst 2004). Whereas ipsilateral HS cells do not seem to be connected to the CNMNs, current injection into the contralateral HSS cell elicited a strong and significant change of the firing frequency in CNMNs, especially in CNMN7 (Fig. 5g). In addition, CNMN7 showed a response to current injection into the contralateral VS2 and VS3 cell, indicating an additional source of visual motion input from the contralateral eye.

From the experiments described above, we conclude that CNMN7 receives visual input from the ipsilateral VS2 and VS3 and from the contralateral HSS. To test whether these two types of inputs are sufficient to explain the visual response properties of the CNMN7, we measured the receptive fields of CNMN6 and CNMN7 by intracellular recordings and compared it with the ones from VS cells and HSS (Fig. 6). The receptive fields of the CNMN6 (Fig. 6a) and CNMN7 (Fig. 6b) are similar to the ones measured extracellularly from the cervical neck nerve (Huston and Krapp 2008). Like VS2 and VS3 (Wertz et al. 2009b; Krapp et al. 1998), the CNMNs respond mainly to frontal vertical downward motion stimuli. The linear superposition of the receptive fields of VS2 and VS3 (Fig. 6c) matches well the measured receptive field of the CNMNs. Surprisingly, the receptive field of HSS (Fig. 6d) cannot be seen in the receptive fields of the CNMNs. 


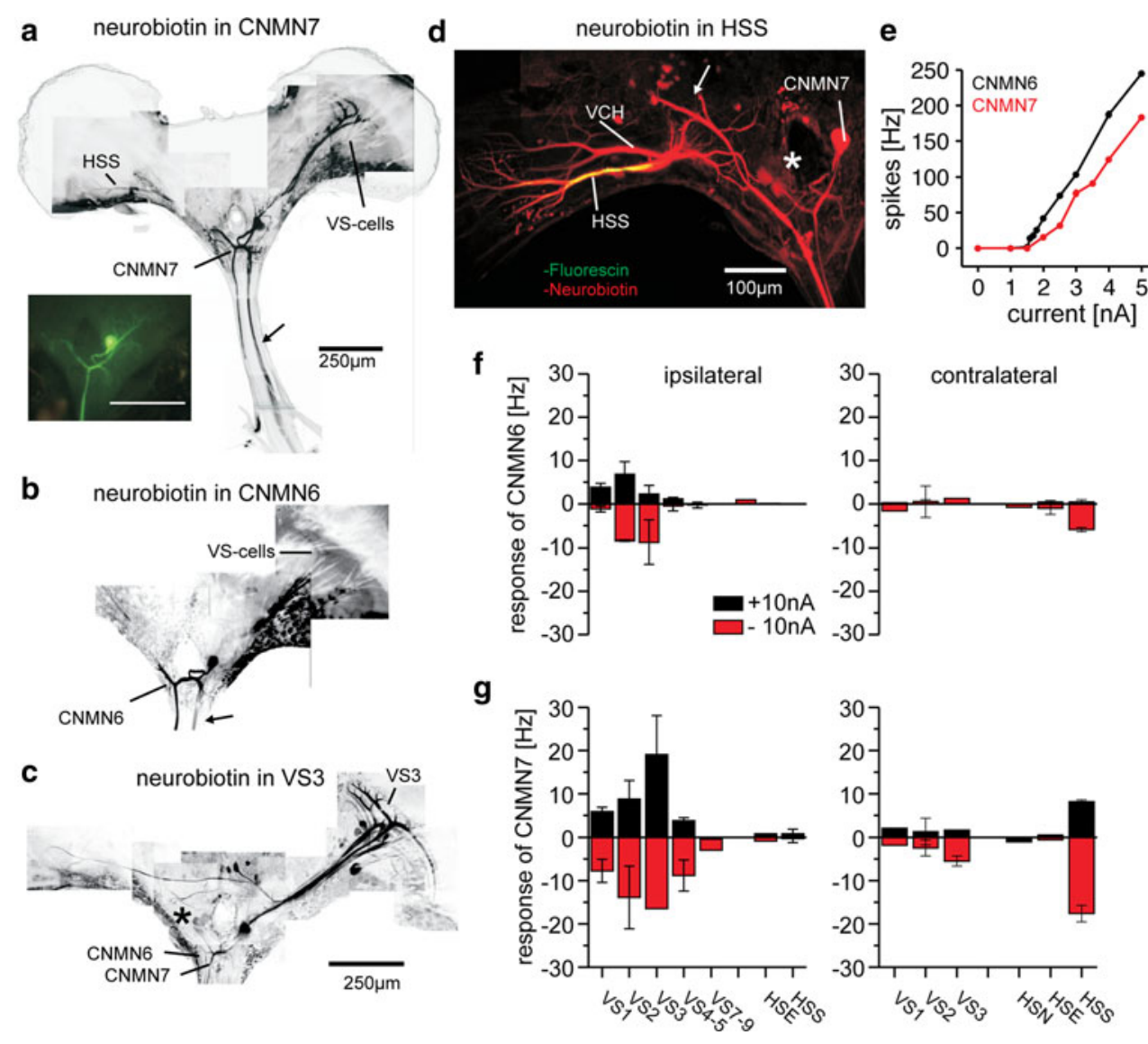

Fig. 5 Connectivity of CNMNs with LPTCs. Dye coupling of CNMNs to VS2/3 and HSS by neurobiotin injections into CNMN7 (a), CNMN6 (b), VS3 (c) and HSS (d). The injected cell was labeled with fluorescein (small picture in a and yellow cell in d). Similar results were achieved by injecting neurobiotin into the following number of cells: CNMN7 $n=4$, CNMN6 $n=2$, VS2 $n=2$, VS3 $n=2$, HSS $n=2$. e CNMN6 and CNMN7 were depolarized by current injections to gain a resting firing frequency. In the example, around $1.5 \mathrm{nA}$ was necessary to elicit spikes in CNMN6 and CNMN7, which had resting membrane potentials of -62 and $-65 \mathrm{mV}$, respectively. Response of CNMN6 (f) and CNMN7 (g) to current injection of $-10 \mathrm{nA}$ (red columns) and $+10 \mathrm{nA}$ (black columns) in different LPTCs ipsilateral (left) and contralateral (right),

However, from the dye coupling and the experiments with current injection, we expect a strong influence of HSS to the visual motion response of CNMN7. To analyze whether the input from VS cells would be sufficient to explain the rotational action field of CNMNs, we calculated the action field as expected from the linear superposition of the action fields of VS2 and VS3 (Fig. 6e). From this action field, we compared the tuning of the expectation with the measured one along the equator. The equatorial tuning expresses the preferred axis of rotation within the horizontal plane. For both CNMN7 and CNMN6 (Fig. 6e), the measured preferred axis of rotation is the pitch axis, whereas the calculated preferred axis (based on the receptive fields) is shifted by about $30^{\circ}$ towards the roll axis. Thus, an respectively. On the ipsilateral side, VS2 and VS3 elicited the strongest response in CNMN6/CNMN7, whereas on the contralateral side HSS influenced the cells most. Although not visible in the dye coupling experiments, the contralateral VS1-VS3 elicited a response in CNMN7. Mean response of CNMN6 (f) to current injection into $n$ cells (ipsilateral: VS $1 n=4, \operatorname{VS} 2 n=2, \operatorname{VS} 3 n=2, \operatorname{VS} 4 / 5 n=5$, VS7-9 $n=3$, HSE $n=1$, HSS $n=1$, contralateral: VS1 $n=1$, $\operatorname{VS} 2 n=2, \operatorname{VS} 3 n=1, \operatorname{HSN} n=1, \operatorname{HSE} n=1, \operatorname{HSS} n=1)$. Mean response of CNMN7 (g) to current injection into $n$ cells (ipsilateral: VS1 $n=3$, VS2 $n=3$, VS $3 n=2$, VS4/5 $n=3$, VS7 $-9 n=1$, HSE $n=1$, HSS $n=3$, contralateral: VS1 $n=1$, VS2 $n=2$, VS3 $n=1, \operatorname{HSN} n=1, \operatorname{HSE} n=1, \operatorname{HSS} n=2$ )

electrical coupling to VS2 and VS3 alone is not sufficient to explain the rotational action field of CNMNs.

To elucidate the influence from the contralateral side, we performed two experiments. First, we measured the response of CNMN7 as a function of the stimulus size (Fig. 7a). The stimulus consisted of the global pitch movement, shown within windows of the following sizes: Window 1: $32^{\circ} \times 48^{\circ}$, Window 2: $96^{\circ} \times 48^{\circ}$, Window 3: $96^{\circ} \times 144^{\circ}$ and Window $496^{\circ} \times 240^{\circ}$ (see Fig. 7a) Whereas the responses of VS cells (here shown for VS2) were readily saturated when the stimulus coincided with their receptive field (stimulus 2), the response of CNMNs (here shown for CNMN7) gradually increased with increasing window size reaching a maximum only when the 


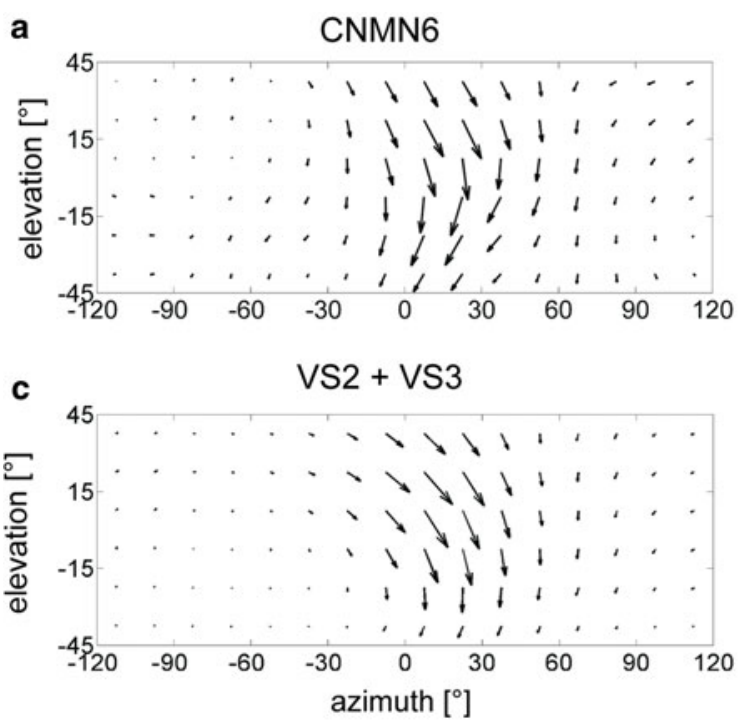

e expected Action Field from CNMN7 RF

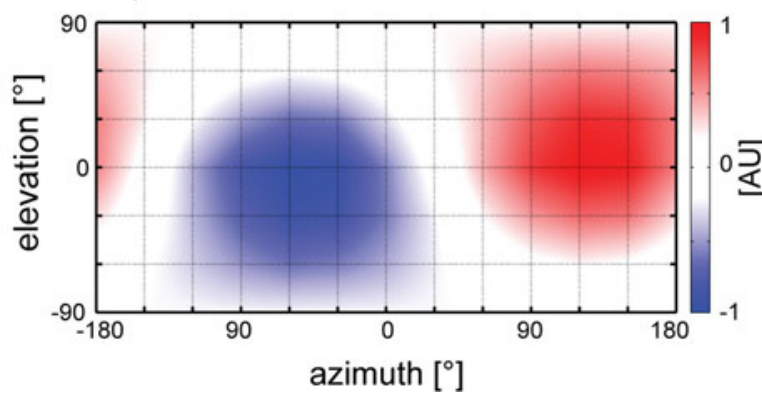

b

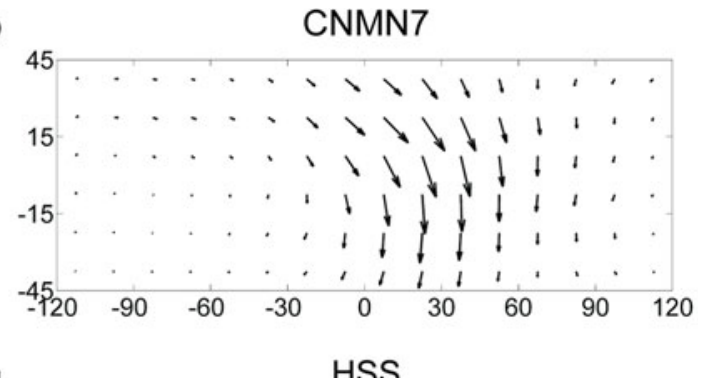

d

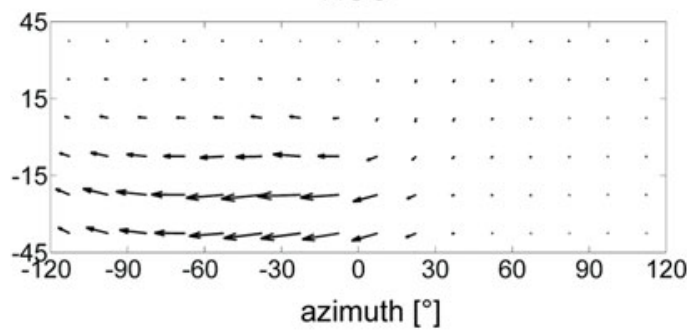

f equatorial Tuning

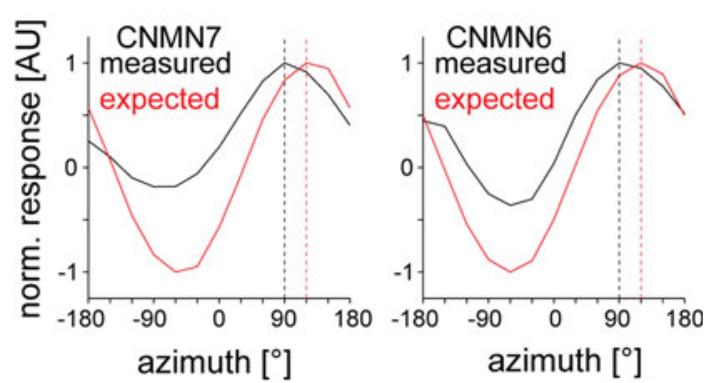

Fig. 6 Receptive fields of CNMN6 and CNMN7. a Mean receptive field of CNMN6 from $n=2$ flies. b Mean receptive field of CNMN7 from $n=6$ flies. Both CNMNs have receptive fields with similarities to optic flows generated during a nose-up pitch movement of the fly. c The receptive fields are similar to the receptive fields of VS2 and VS3 (c data from Wertz et al. 2009a, b). d Mean receptive field of

stimulus was presented in its full size. Accordingly, the response of CNMN7 to a pitch movement was only $50 \%$ of the maximal value when the contralateral eye was occluded (Fig. 7b). Both results emphasize again a binocular integration of visual motion information that is not obvious in the receptive fields of the cells, as shown in Fig. 6a. Although the contralateral dendrite of both CNMNs is not located next to the axon terminal of contralateral VS cells, current injection into contralateral VS cells elicited a spike frequency change in CNMN7. Thus, the current injection should be transmitted indirectly through an additional cell. To test whether the contralateral CNMNs are connected to the ipsilateral CNMNs, we performed double recordings of these cells (Fig. 7c). We injected current into the cell body of the ipsilateral CNMN7 and measured the response in the ipsilateral CNMN6, the contralateral CNMN6 and the contralateral CNMN7. We found a strong ipsilateral connectivity between CNMN6 and CNMN7 and, in addition, a connectivity between CNMN7s of either side. This result is
HSS from $n=4$ flies. Although expected from the connectivity, the motion response of HSS is not observable in the receptive field of CNMNs. e Linear predicted action field for CNMN7 from its receptive field. $\mathbf{f}$ The equatorial tuning indicates the shift between the measured and expected action fields of CNMN7 as well as CNMN6

confirmed by the neurobiotin labeling where the contralateral cell bodies are slightly stained after neurobiotin injection into CNMN7, VS cells or HSS (e.g., in Fig. 5d indicated by an asterisk).

To answer the question if both eyes are necessary for gaze stabilization in flies, we determined the fly's compensatory head movements around the pitch axis either with both eyes intact or with one eye covered. Therefore, flies were facing the LED arena (Fig. 8a) on which the stimulus movie was presented. The head of the fly was filmed from behind. To simplify the tracking of the head movements off-line, the head was labeled with two white dots (see Fig. 8b). An example movie sequence of a head declination is shown in Fig. 8b. The pitch stimulus was shown with a sinusoidal velocity (Fig. 8c). Having both eyes intact, the fly pitches down and up its head following the stimulus (Fig. 8c, black trace). However, with one eye occluded, no stimulus-induced pitch movements could be observed (Fig. 8c, red trace). Thus, both eyes are necessary 

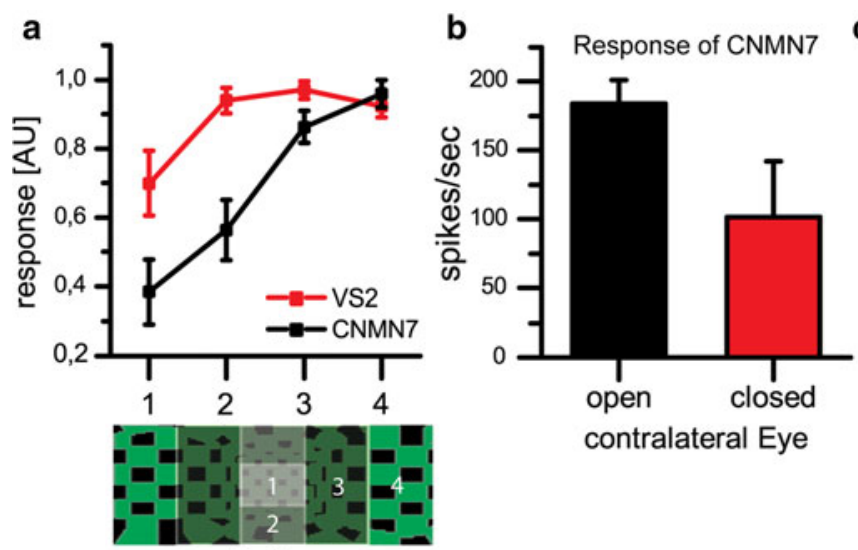

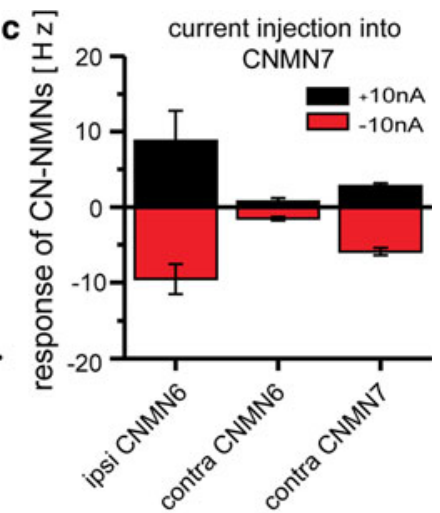

Pattern

Fig. 7 Influence from contralateral side. a Mean responses \pm SEM of CNMN7 ( $n=4$ flies) and VS2 ( $n=4$ flies) to an increasing noseup pitch movement. The strongest response was elicited in VS2 with a pitch movement covering the receptive field of the cell, whereas CNMN7 increases its firing rate to broader stimuli. b Response of CNMN7 to pitch stimulus with both eyes open (black) and the
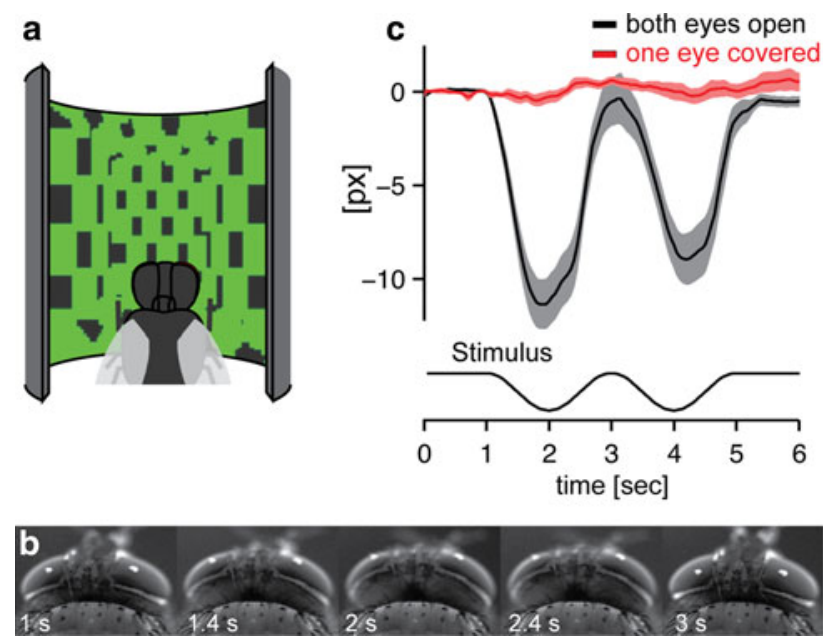

Fig. 8 Optomotor response of the fly head. a Schematic drawing of the stimulus presentation. The head of the fly could freely move, while a nose-up and nose-down pitch movement was presented with sinusoidal velocity. b Sequential images of the head of the fly with time intervals of $300 \mathrm{~ms}$ showing the pitch movement of the head. To calculate the vertical and horizontal movement, the head was labeled with two white dots. c Compensatory head response with both eyes open (black trace) and one eye occluded (red). Mean response \pm SD of seven flies

to induce optomotor pitch movements of the head. In addition, the behavior reveals an all or none characteristiceither the fly moves its head or does not.

Recent experiments revealed an integration of sensory stimuli from different modalities at the level of neck motor neurons of the frontal and ventral cervical nerve (Huston and Krapp 2009; Haag et al. 2010). It has been demonstrated that these neurons receive not only visual input, but, in addition, input from the campaniform sensilla of the halteres, the contralateral eye closed (red). c Responses of CNMNs to current injection of $-10 \mathrm{nA}$ (red columns) and $+10 \mathrm{nA}$ (black columns) into the soma of CNMN7. Current injection elicited a response in the ipsilateral CNMN6 and the contralateral CNMN7. Mean response of ipsilateral CNMN6 $(n=4)$, contralateral CNMN6 $(n=2)$ and contralateral CNMN7 $(n=3)$

wind-sensitive Johnston organ on the antennae and a central neuron reflecting locomotor activity. In order to investigate whether motor neurons of the cervical nerve also receive multimodal sensory input, we performed a series of experiments. In a first set of experiments, we recorded extracellularly from the cervical nerve via a suction electrode (Fig. 9a, b). The wind puff directed to the abdomen of the fly elicited fast haltere beating (Fig. 9a, red trace). The haltere beating is accompanied by a strong increase in activity of the cervical nerve (Fig. 9a, black trace). Note that the wind puff to the abdomen itself did not elicit a response in the neck motor neurons if the haltere beating was not triggered. Figure $9 \mathrm{~b}$ shows the response of the cervical nerve to a wind puff directed to the antennae of the fly. Like the active haltere beating, the stimulation of the antennae elicited strong activity in the cervical nerve. Due to the strong increase of activity of several units of the cervical nerve during these two stimuli, we were not able to identify the recorded neurons. Therefore, we repeated the experiments, but recorded intracellularly from CNMN6 and 7. Figure 9c, d shows the results for intracellular recording of CNMN6 and Fig. 9e, $f$ for CNMN7. Both neurons respond with a strong increase in firing frequency to the beating of the halteres. The wind stimulus directed to the antennae elicited only one or a few action potentials in these neurons. These results demonstrate that, as neck motor neurons of the frontal (Huston and Krapp 2009) and the ventral cervical nerve (Haag et al. 2010), CNMNs also perform multisensory integration.

\section{Discussion}

In this study, we described a neural circuit involved in the head declination of the fly. Our ego-motion tuning 
experiments confirmed the assumption by anatomical findings (Strausfeld et al. 1987) that CNMN6 and CNMN7 are involved in the head pitch movement. The double recordings and dye coupling experiments revealed a complex connectivity of CNMN6 and 7 to visual interneurons of the ipsilateral and the contralateral lobula plate as well as among each other. In addition, we demonstrated in our behavioral experiment the need of a binocular integration to activate an optomotor pitch response of the head. In the following, we will discuss the evidence for this circuitry as well as the visuomotor transformation executed by it, the multisensory integration in CNMNs and the behavioral relevance of a head declination.

\section{Visuomotor transformation}

In the fly, motion vision is processed by the lobula plate tangential cells (Borst and Euler 2011; Borst et al. 2010), which receive visual motion information via different processing channels from the photoreceptors on the retina (Meinertzhagen and O'Neil 1991; Joesch et al. 2010). With a series of double recordings and neurobiotin injections, we showed that CNMN7 receives input from two groups of tangential cells, namely VS2 and VS3 (VS2/3) ipsilateral and HSS contralateral. Whereas the impact from VS2 and VS3 is observable in the receptive field of CNMN7, the impact of HSS is not (Fig. 6). This may be due to the fact that our ventral motion stimulus was limited in its spatial extent to $-45^{\circ}$ in elevation. The local motion sensitivity of HSS was previously shown to be maximally around $40^{\circ}$ and the receptive field extends all the way down to the ventral pole (Taylor and Krapp 2007). However, the receptive field of a not further identified CNMN measured extracellularly by Huston and Krapp (2008) is similar to the receptive fields of CNMN6 and CNMN7, but also does not show a response to horizontal motion at $-70^{\circ}$. Therefore it is unlikely that the impact of HSS would be observable in the CNMN7 receptive field with a ventrally more extended stimulus device. Another explanation for local directional preferences of the HSS cell not being reflected in the CNMN7 receptive field may be due to a low synaptic gain in between the HSS cell and the CNMN7 upon local opposed to motion stimuli.

In addition to the input from lobula plate tangential cells, we found a connectivity of CNMN7 to the ipsilateral CNMN6 and to the contralateral CNMN7 (illustrated in Fig. 10). Whether CNMN6 and CNMN7 are directly coupled, or indirectly via the VS cells, providing input to both of them is hard to determine. A previous study demonstrated that the amount of neurobiotin found in other cells due to the dye coupling correlates with the coupling strength (Haag and Borst 2005). On injecting neurobiotin in CNMN7, CNMN6 is much less stained than VS cells and vice versa, which can indicate either an indirect coupling or a weak direct coupling between the CNMNs. The dye coupling found between CNMN7 of either side suggests a direct connection between these cells. Through this coupling, CNMN7 could integrate visual motion information from VS cells of the contralateral side. Thus, in addition to motion information from the contralateral horizontal system, via HSS, CNMN7 integrates motion information from the vertical system via the contralateral CNMN7. In agreement with this, the response of CNMN7 increased with increasing size of the visual stimulus (Fig. 7a) and decreased when covering the contralateral eye (Fig. 7b).

To identify the cells providing synaptic input to CNMN7, we injected current into candidate neurons of the lobula plate while depolarizing CNMN7. Under these conditions, current injection into VS2/3 had the strongest influence on the firing rate of CNMN7. As VS2/3 are also strongly co-labeled after neurobiotin injection into CNMNs, we suggest that VS2/3 provide the strongest input to CNMN7. In agreement with this, CNMN7 responded only slightly on covering the ipsilateral eye. Covering the contralateral eye decreased the response of CNMN7 by $45 \%$. These two experiments demonstrate that (1) ipsilateral visual motion is necessary to elicit a strong response in CNMN7, most likely via VS2/3, and (2) this response is further enhanced by input from the contralateral eye in a nonlinear way. How can this finding explain that no optomotor pitch response was observable when one eye was covered? Let us assume that for head declination, each CNMN7 provides $50 \%$. Covering the left eye silences the left CNMN7 completely and decreases the responses of the right CNMN7 to about $50 \%$. This results in an overall activation level of $25 \%$ of the activation in the unimpaired fly. Obviously, this reduced activity is insufficient to elicit the head pitch response. Whether this loss of function is due to the fact that the lower firing frequency of CNMN7 does not lead to a tonic muscle contraction or the simultaneous contraction of muscles of both sides is necessary needs to be resolved. However, integrating binocular motion information increases not only the cell's preference for rotational optic flow, like it was shown for neck motor neurons receiving indirect input from lobula plate tangential cells (Huston and Krapp 2008), but it also triggers the motor response. The described neural circuit accomplishes the required binocular integration by connecting the CNMN7's from both hemispheres to each other.

\section{Multisensory integration}

To monitor ego-motion, flies use the visual system as well as their halteres (Hengstenberg 1991). Halteres are small club-shaped appendages oscillating during flight at the 
Fig. 9 Responses of cervical nerve motor neurons during haltere beating $(\mathbf{a}, \mathbf{c}, \mathbf{e})$ and wind stimuli (b, d, f).

a Simultaneously recorded activity in the cervical nerve (black trace) and vertical position of the haltere (red trace). During active beating of the halteres, a strong increase in spike activity is observed. Same data have been obtained from recordings of two more flies. c Intracellular recording from the dendrite of CNMN6. Beating of the halteres elicited a strong depolarization and the generation of action potentials in CNMN6. The same data were obtained from five more flies. e Intracellular recording from the soma of CNMN7. Same data were obtained from three more flies. b Suction electrode recording of the cervical nerve to a wind puff (gray bar) directed toward the antennae. The nerve responded with an increase of activity.

d Intracellular recording from the dendrite of CNMN6. The wind puff elicited only one or a few spikes. Same data were obtained from three more flies. f Intracellular recording from the dendrite of CNMN7. Same data were obtained from two more flies
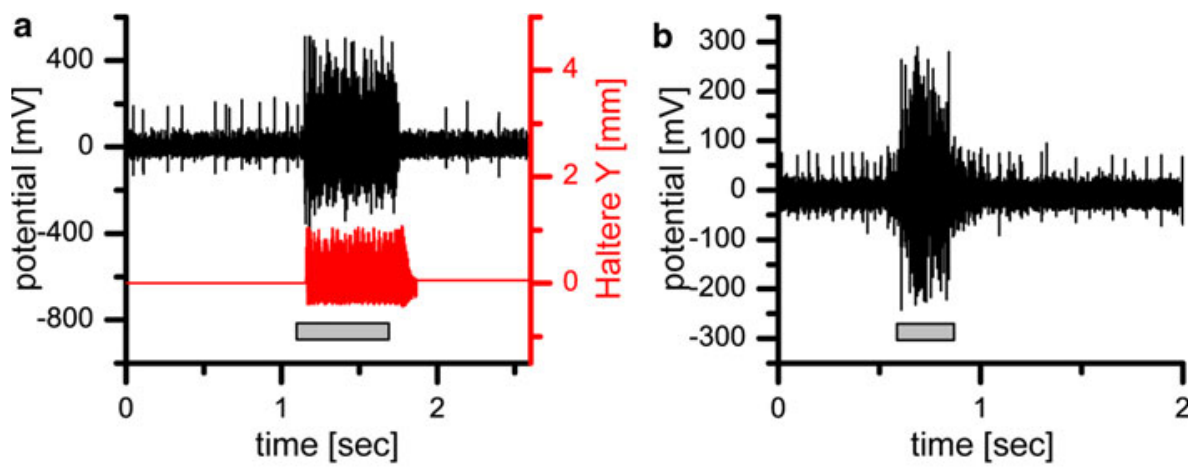

c

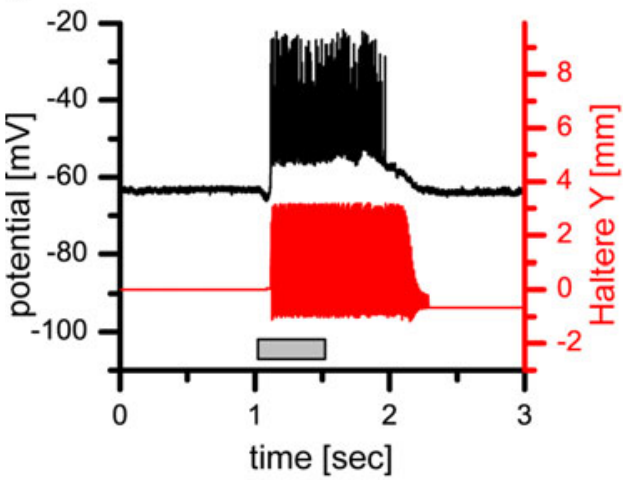

d
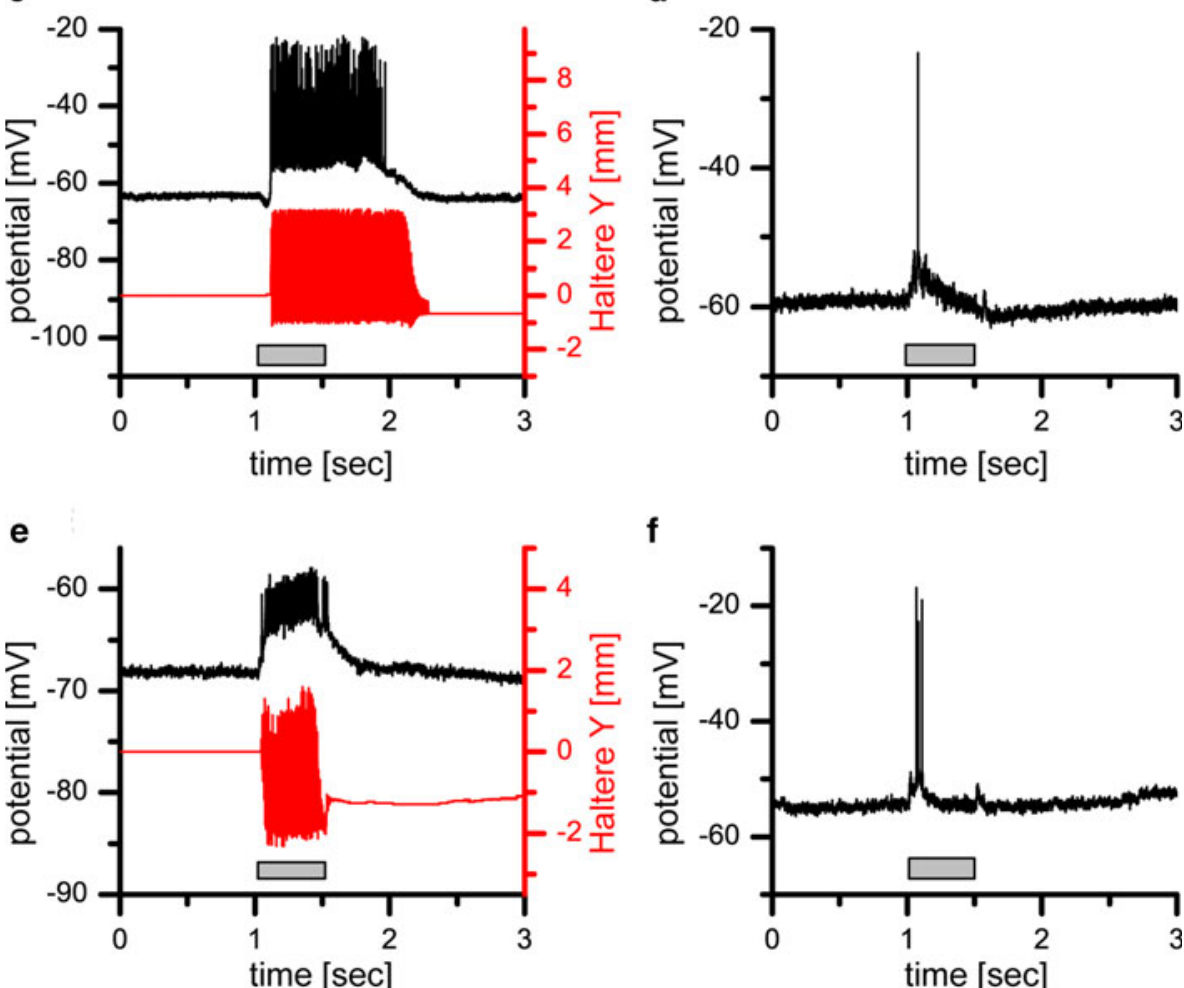

f

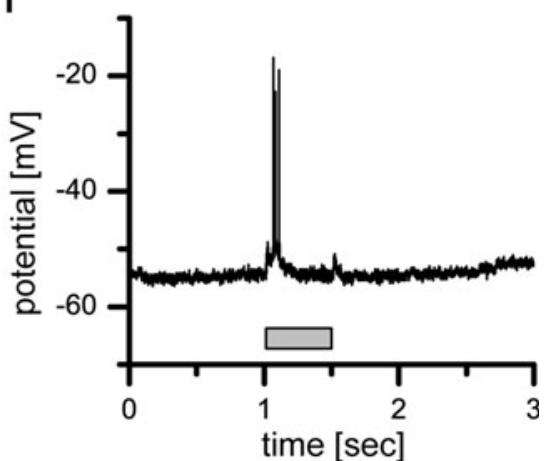

same frequency as the wings, but in antiphase. They detect the coriolis forces imposed upon them by rotations of the fly via a field of sensillae at the base of the halteres (Nalbach and Hengstenberg 1994; Dickinson 1999; Fox and Daniel 2008; Pringle 1948). The lobula plate tangential cells are more sensitive to comparatively slow changes of rotation velocities of the fly, whereas the halteres are more sensitive to faster accelerations (Sherman and Dickinson 2003; Hengstenberg 1991). Neck motor neurons are known to integrate both visual and haltere inputs (Sandeman and Markl 1980). Furthermore, it has been shown that neck motor neurons from the frontal and the ventral cervical nerve do not produce action potentials in response to visual stimuli alone, but only if the halteres are concurrently beating (Huston and Krapp 2009). From these experiments, it has been suggested that the input from the halteres depolarize the neck motor neuron above action potential threshold and the firing rate is then modulated by visual input (Haag et al. 2010). In our experiments, we also found that both CNMN6 and 7 responded strongly to the movements of the halteres (Fig. 9c, e). However, in contrast to the experiments on the frontal and ventral cervical nerve, we found as expected from previous studies of the whole nerve (Milde et al. 1987; Huston and Krapp 2009) that CNMN6 and 7 responded to global motion stimuli with an increase in firing rate without additional sensory input. This might be due to the larger size of the visual stimulus used in this study. Visual stimuli of smaller sizes failed also to elicit action potentials in CNMNs.

In addition to input from the visual system and the halteres, insect neck motor neurons receive input from the proprioceptive prosternal organ (Preuss and Hengstenberg 1992; Paulk and Gilbert 2006), from the wind-sensitive antennae (Haag et al. 2010), the ocelli (Hung et al. 2011) 


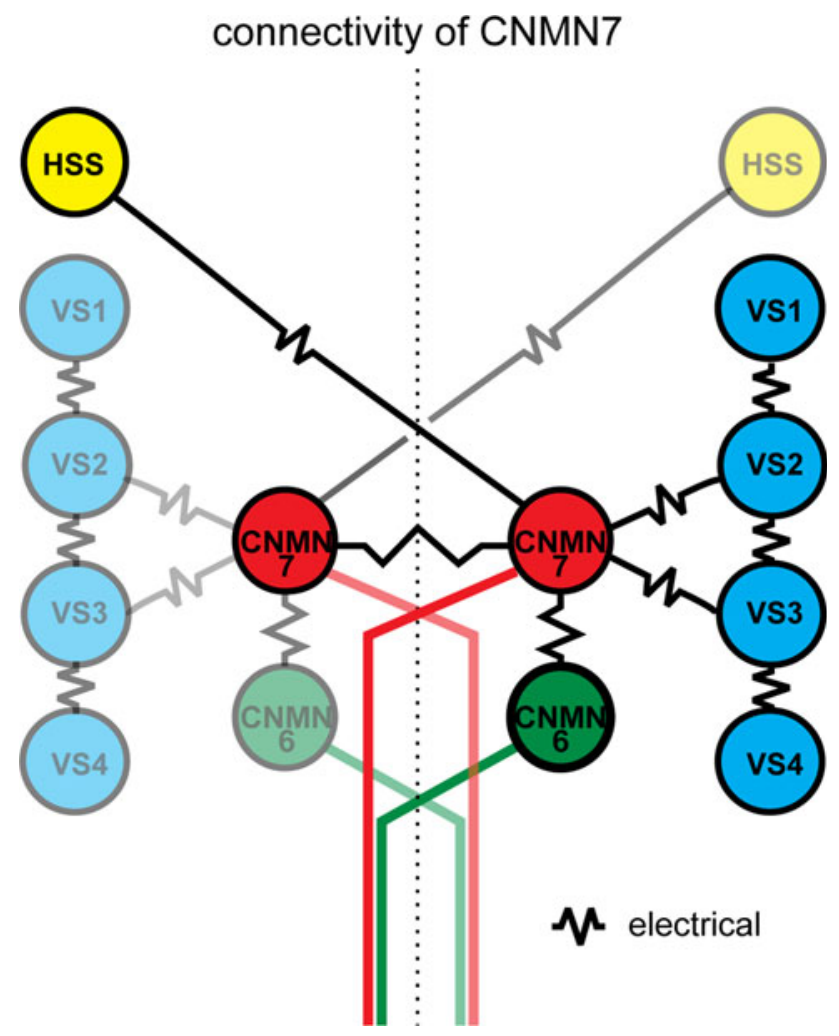

Fig. 10 Schematic of the investigated neural circuit. CNMN7 is electrical coupled ipsilaterally to VS2 and VS3 (blue) and to CNMN6 (green) and contralaterally to HSS (yellow) and CNMN7 (slight red)

and a central gating neuron (Haag et al. 2010; Rosner et al. 2009). Especially the input from the ocelli could be very interesting as it is shown that VS neurons in the blowfly lobula plate receive short latency ocellar signals that code rotations of the head about horizontal axes (Parsons et al. 2010). Although the ocelli are thought to encode different axes $\left(0^{\circ}, 45^{\circ}\right.$ and $\left.-45^{\circ}\right)$ than the pitch axis $\left(90^{\circ}\right)$, it could be still very interesting if and how the ocellar input changes the tuning of CNMNs. Whether the responses of CNMN6 and 7 indicate sensory input from the campaniform sensilla of the halteres or input from a central neuron reflecting the behavioral state of the fly needs to be clarified by further experiments. However, the neurobiotin experiments revealed at least one ascending cell connected to CNMNs (see Fig. 5), which might reflect input from the haltere nerve.

\section{State dependency of CNMN6 and CNMN7}

Hengstenberg (1991) found an optomotor head response of the fly only when the halteres were beating. Therefore, he concluded that blowflies make visually induced gaze-stabilizing head movements only when walking or flying, and not when standing still (Hengstenberg 1991). Along these lines, Rosner et al. (2009) found that the gain of pitch head movements depended strongly on the internal state of the fly. When the flies were in a low activity state, i.e., the halteres did not move, visual stimulation elicited only small deflections of the head. If, in contrast, the flies were in a high activity state, indicated by haltere beating, the gain of the pitch response was high. From the fact that removal of the halteres did not prevent the high activity state, the authors concluded that the source of the signal was from the central nervous system and not due to reafferences of the haltere system (Rosner et al. 2009). The same conclusions were drawn from experiments on neck motor neurons of the ventral cervical nerve. In that study, both the time course of the excitation and the results of fixating the halteres speak in favor of a central signal influencing the responsiveness of neck motor neurons to visual motion stimuli (Haag et al. 2010). In addition, statedependent responses of lobula plate tangential cells were shown recently in Drosophila (Maimon et al. 2010; Chiappe et al. 2010) as well as in Calliphora (Rosner et al. 2010; Jung et al. 2011). From all these findings, we expect the responses of CNMN6/7 to be state dependent. A possible candidate that mediates this change might be the neuromodulator octopamine. Octopamine has been shown to change the behavioral state of an animal (Stevenson et al. 2005) and is known to increase the response to visual stimuli in the lobula plate of flies (Longden and Krapp 2009, 2010). Thus, additional experiments with octopamine could verify if and how central activity affects the responsiveness of these CNMNs.

Acknowledgments We are grateful to Renate Gleich and Ursula Weber for excellent technical assistance. This work was supported by the Max-Planck-Society and a grant of the BMBF to the Bernstein Center for Computational Neuroscience (BCCN) Munich to A.B.

\section{References}

Borst A, Haag J, Reiff DF (2010) Fly motion vision. Annu Rev Neurosci 33:49-70. doi:10.1146/annurev-neuro-060909-153155

Borst A, Euler T (2011) Seeing things in motion: models, circuits, and mechanisms. Neuron 71:974-994. doi:10.1016/j.neuron.2011.08. 031

Borst A, Weber F (2011) Neural action fields for optic flow based navigation: a simulation study of the fly lobula plate network. PLoS One 6:e16303. doi:10.1371/journal.pone.0016303

Chiappe ME, Seelig JD, Reiser MB, Jayaraman V (2010) Walking modulates speed sensitivity in Drosophila motion vision. Curr Biol 20:1470-1475. doi:10.1016/j.cub.2010.06.072

Denk W, Strickler J, Webb W (1990) Two-photon laser scanning fluorescence microscopy. Science 248:73-76. doi:10.1126/science. 2321027

Dickinson MH (1999) Haltere-mediated equilibrium reflexes of the fruit fly, Drosophila melanogaster. Philos Trans R Soc Lond B Biol Sci 354:903-916. doi:10.1098/rstb.1999.0442

Fox J, Daniel T (2008) A neural basis for gyroscopic force measurement in the halteres of Holorusia. J Comp Physiol A 194:887-897. doi:10.1007/s00359-008-0361-z 
Geiger G, Poggio T (1977) On head and body movements of flying flies. Biol Cybern 25:177-180. doi:10.1007/bf00365214

Haag J, Borst A (2004) Neural mechanism underlying complex receptive field properties of motion-sensitive interneurons. Nat Neurosci 7:628-634. doi:10.1038/nn1245

Haag J, Denk W, Borst A (2004) Fly motion vision is based on Reichardt detectors regardless of the signal-to-noise ratio. Proc Natl Acad Sci 101:16333-16338. doi:10.1073/pnas.0407368101

Haag J, Borst A (2005) Dye-coupling visualizes networks of largefield motion-sensitive neurons in the fly. J Comp Physiol A 191:445-454. doi:10.1007/s00359-005-0605-0

Haag J, Wertz A, Borst A (2010) Central gating of fly optomotor response. Proc Natl Acad Sci 107:20104-20109. doi:10.1073/ pnas. 1009381107

Hengstenberg R (1972) Eye movements in the housefly Musca domestica. Datenverarbeitung im visuellen System der Arthropoden. Springer, Berlin

Hengstenberg R (1991) Gaze control in the blowfly Calliphora: a multisensory, two-stage integration process. Sem Neurosci 3:19-29. doi:10.1016/1044-5765(91)90063-t

Hung YS, van Kleef J, Ibbotson M (2011) Visual response properties of neck motor neurons in the honeybee. J Comp Physiol A 197: 1173-1187. doi:10.1007/s00359-011-0679-9

Huston SJ, Krapp HG (2008) Visuomotor transformation in the fly gaze stabilization system. PLoS Biol 6:e173. doi:10.1371/journal. pbio.0060173

Huston SJ, Krapp HG (2009) Nonlinear integration of visual and haltere inputs in fly neck motor neurons. J Neurosci 29:13097-13105. doi:10.1523/jneurosci.2915-09.2009

Joesch M, Plett J, Borst A, Reiff DF (2008) Response properties of motion-sensitive visual interneurons in the lobula plate of Drosophila melanogaster. Curr Biol 18:368-374. doi:10.1016/ j.cub.2008.02.022

Joesch M, Schnell B, Raghu SV, Reiff DF, Borst A (2010) ON and OFF pathways in Drosophila motion vision. Nature 468:300 304. doi:10.1038/nature09545

Jung SN, Borst A, Haag J (2011) Flight activity alters velocity tuning of fly motion-sensitive neurons. J Neurosci 31:9231-9237. doi:10.1523/jneurosci.1138-11.2011

Karmeier K, Krapp HG, Egelhaaf M (2003) Robustness of the tuning of fly visual interneurons to rotatory optic flow. J Neurophysiol 90:1626-1634. doi:10.1152/jn.00234.2003

Krapp HG, Hengstenberg R (1996) Estimation of self-motion by optic flow processing in single visual interneurons. Nature 384: 463-466. doi:10.1038/384463a0

Krapp HG, Hengstenberg B, Hengstenberg R (1998) Dendritic structure and receptive-field organization of optic flow processing interneurons in the fly. J Neurophysiol 79:1902-1917

Longden KD, Krapp HG (2009) State-dependent performance of optic-flow processing interneurons. J Neurophysiol 102:36063618. doi:10.1152/jn.00395.2009

Longden KD, Krapp HG (2010) Octopaminergic modulation of temporal frequency coding in an identified optic flow-processing interneuron. Front Syst Neurosci 4. doi:10.3389/fnsys.2010. 00153

Maimon G, Straw AD, Dickinson MH (2010) Active flight increases the gain of visual motion processing in Drosophila. Nat Neurosci 13:393-399. doi:10.1038/nn.2492

Meinertzhagen IA, O'Neil SD (1991) Synaptic organization of columnar elements in the lamina of the wild type in Drosophila melanogaster. J Comp Neurol 305:232-263. doi:10.1002/cne. 903050206

Milde JJ, Seyan HS, Strausfeld NJ (1987) The neck motor system of the fly Calliphora erythrocephala. 2. Sensory organization. J Comp Physiol A 160:225-238
Nalbach G, Hengstenberg R (1994) The halteres of the blowfly Calliphora. J Comp Physiol A 175:695-708. doi:10.1007/bf0019 1842

Nordström K, Barnett PD, Moyer de Miguel IM, Brinkworth RSA, O'Carroll DC (2008) Sexual dimorphism in the hoverfly motion vision pathway. Curr Biol 18:661-667. doi:10.1016/j.cub.2008. 03.061

Parsons MM, Krapp HG, Laughlin SB (2010) Sensor fusion in identified visual interneurons. Curr Biol 20:624-628. doi:10.1016/j.cub. 2010.01.064

Paulk A, Gilbert C (2006) Proprioceptive encoding of head position in the black soldier fly, Hermetia illucens (L.) (Stratiomyidae). J Exp Biol 209:3913-3924. doi:10.1242/jeb.02438

Preuss T, Hengstenberg R (1992) Structure and kinematics of the prosternal organs and their influence on head position in the blowfly Calliphora erythrocephala. J Comp Physiol A 171:483-493. doi:10.1007/bf00194581

Pringle JWS (1948) The gyroscopic mechanism of the halteres of diptera. Philos Trans R Soc Lond B Biol Sci 233:347-384. doi: 10.1098/rstb.1948.0007

Quiroga RQ, Nadasdy Z, Ben-Shaul Y (2004) Unsupervised spike detection and sorting with wavelets and superparamagnetic clustering. Neural Comput 16:1661-1687. doi:10.1162/089976 604774201631

Rosner R, Egelhaaf M, Grewe J, Warzecha AK (2009) Variability of blowfly head optomotor responses. J Exp Biol 212:1170-1184. doi:10.1242/jeb.027060

Rosner R, Egelhaaf M, Warzecha A-K (2010) Behavioural state affects motion-sensitive neurones in the fly visual system. J Exp Biol 213:331-338. doi:10.1242/jeb.035386

Sandeman DC, Markl H (1980) Head movements in flies (Calliphora) produced by deflection of the halteres. J Exp Biol 85:43-60

Sherman A, Dickinson MH (2003) A comparison of visual and haltere-mediated equilibrium reflexes in the fruit fly Drosophila melanogaster. J Exp Biol 206:295-302. doi:10.1242/jeb.00075

Srinivasan MV, Zhang SW (2004) Visual motor computations in insects. Annu Rev Neurosci 27:679-696. doi:10.1146/Annurev. Neuro.27.070203.144343

Stevenson PA, Dyakonova V, Rillich J, Schildberger K (2005) Octopamine and experience-dependent modulation of aggression in crickets. J Neurosci 25:1431-1441. doi:10.1523/jneurosci. 4258-04.2005

Strausfeld NJ (1976) Atlas of an insect brain. Springer, New York

Strausfeld NJ, Seyan HS (1985) Convergence of visual, haltere, and prosternal inputs at neck motor neurons of Calliphora erythrocephala. Cell Tissue Res 240:601-615. doi:10.1007/bf00216350

Strausfeld NJ, Seyan HS, Milde JJ (1987) The neck motor system of the fly Calliphora erythrocephala. 1. Muscles and motor neurons. J Comp Physiol A 160:205-224

Strausfeld NJ, Gronenberg W (1990) Descending neurons supplying the neck and flight motor of diptera: organization and neuroanatomical relationships with visual pathways. J Comp Neurol 302:954-972. doi:10.1002/cne.903020419

Taylor GK, Krapp HG (2007) Sensory systems and flight stability: what do insects measure and why? Adv Insect Physiol Insect Mech Control 34:231-316. doi:10.1016/S0065-2806(07)34005-8

Wertz A, Borst A, Haag J (2008) Nonlinear integration of binocular optic flow by DNOVS2, a descending neuron of the fly. J Neurosci 28:3131-3140. doi:10.1523/jneurosci.5460-07.2008

Wertz A, Gaub B, Plett J, Haag J, Borst A (2009a) Robust coding of ego-motion in descending neurons of the fly. J Neurosci 29:14993-15000. doi:10.1523/jneurosci.3786-09.2009

Wertz A, Haag J, Borst A (2009b) Local and global motion preferences in descending neurons of the fly. J Comp Physiol A 195:1107-1120. doi:10.1007/s00359-009-0481-0 